\title{
Crisis: un área de oportunidad para retomar y proponer nuevos paradigmas
}

\author{
Crisis: an area of opportunity to retake and propose new paradigms
}

\section{David Santiago-Germán ${ }^{*}$}

División de Investigación en Salud, Unidad Médica de Alta Especialidad (UMAE) de Traumatología, Ortopedia y Rehabilitación "Dr. Victorio de la Fuente Narváez"Instituto Mexicano del Seguro Social (IMSS), Ciudad de México, México

La ciencia tiene como propósito principal generar conocimiento nuevo del mundo físico, y es la curiosidad la fuerza fundamental que la motiva ${ }^{1}$. La rama de la filosofía que estudia el conocimiento y cómo se obtiene se conoce como epistemología ${ }^{2}$. Existen diferentes interpretaciones del mundo físico o realidad, sin embargo, en lo que están de acuerdo diversos filósofos y físicos es que hay un mundo, previo a e independiente de nuestra existencia, más allá de nuestra percepción y/o representación de esta ${ }^{3}$. La rama de la filosofía que estudia la naturaleza y la estructura de la realidad se llama ontología ${ }^{4}$. La investigación científica tal y como la conocemos hoy día implica la construcción sistemática de un modelo o paradigma que explique el fenómeno natural de estudio, descrito o representado en un lenguaje universal o matemático, hasta que un nuevo paradigma lo sustituya generando un avance científico ${ }^{5}$.

Una crisis es un cambio profundo de consecuencias importantes en una situación determinada, pero también es un área de oportunidad para generar nuevos paradigmas a partir de hilos de continuidad del conocimiento ya existentes. Un ejemplo de esto es el resultado de la investigación por William Firth Wells en 1934 The Wells evaporation falling curve of droplets, sobre la relación entre el tamaño de las gotas de saliva y su tasa de caída, que ha ayudado a profundizar en la comprensión de la transmisión aérea de las enfermedades infecciosas respiratorias, y cuyo modelo ha sido retomado y mejorado en los últimos 87 años ${ }^{6}$. Otro ejemplo es el primer modelo de transmisión de enfermedad por gotitas de saliva propuesto por el bacteriólogo alemán Carl Flügge en 1897, cuyo hallazgo fue clave para el desarrollo del predecesor de la primera mascarilla quirúrgica por Johann von Mikulicz Radecki el mismo año ${ }^{7}$. Estos tres personajes, entre otros, sentaron las bases de los mecanismos de transmisión de las enfermedades infecciosas respiratorias y del equipo de protección personal. No obstante, la emergencia de una brecha en el conocimiento y la urgencia por llenarla, como en la presente crisis sanitaria por coronavirus 2 del síndrome respiratorio agudo grave (SARSCoV-2), no debe orillarnos a la generación de conclusiones basadas en la ausencia del conocimiento, es decir, en la ignorancia o argumentum ad ignorantiam, lo que se interpreta como «la ausencia de prueba no es prueba de ausencia». Es necesario retomar con urgencia los hilos de continuidad del conocimiento y proponer nuevos modelos que se adapten a las condiciones actuales en el contexto sanitario, económico, educativo, político, social y cultural.

En el presente número de la revista, el lector encontrará tres artículos de revisión. El primero de ellos es la secuela del artículo titulado Embolia Pulmonar en el

\section{Correspondencia}

*David Santiago-Germán

E-mail: david.santiago.german@gmail.com
Disponible en internet: 03-12-2020

Fecha de recepción: 18-11-2020

Fecha de aceptación: 20-11-2020 DOI: 10.24875/REIE.M20000008
Rev Educ Investig Emer. 2019;1(4):124-125 www.medicinadeemergencias.com

Este es un artículo open access bajo la licencia CC BY-NC-ND (http://creativecommons.org/licenses/by-nc-nd/4.0/). 
Servicio de Emergencias, en esta segunda parte los autores abordan las escalas pronósticas y tratamiento. El segundo artículo, titulado Introducción a la Simulación Clínica, es sin duda una herramienta que permitirá al lector familiarizarse con los fundamentos de la simulación, la cual en el contexto actual es ya un nuevo paradigma educativo que permitirá mejorar la seguridad del paciente y acortar la curva de aprendizaje del médico en formación. El último artículo es el segundo de una serie de tres manuscritos sobre la terapia de reanimación hídrica (previamente se trataron los diferentes tipos de líquidos y sus efectos adversos); el actual artículo profundiza en la respuesta fisiológica del cuerpo humano a la administración de líquidos intravenosos.

Por último, la Sociedad Mexicana de Medicina de Emergencia y el Comité Editorial expresamos nuestro agradecimiento y reconocimiento al personal de salud interdisciplinario y transdisciplinario por la labor que realizan diariamente en pro de la presente emergencia sanitaria por SARS-CoV-2.

\section{Bibliografía}

1. Eflin JT. The nature of science: A perspective from the philosophy of science. J Res Sci Teach. 1999;36(1):107-16.

2. Fitzgerald J. Epistemology and reading. Read Res Q. 1996;31(1):36-60.

3. Saridakis E. Information, reality, and modern physics. Int Stud Philos Sci. 2016;30(4):327-41.

4. Guarino N, Oberle D, Staab S. What is an ontology? En: Staab S, Studer R, editores. Handbook on ontologies. International Handbooks on Information Systems. Springer, Berlin, Heidelberg; 2009.

5. Graña-Aramburú A. Filósofos que contribuyeron al progreso de la medicina. Acta Med Per. 2015;32(1):41-9.

6. Xie X, Li Y, Chwang ATY, Ho PL, Seto WH. How far droplets can move in indoor environments - revisiting the Wells evaporation - falling curve. Indoor Air. 2007;17:211-25.

7. Gandhi JA, Churiwala J, Shinde PH, Chaudhari SN. Surgical Mask - the Saviour: from its invention to the COVID-19 Era. Indian J Surg. 2020;12:1-2. 